\title{
MODELLING THE INTRODUCTION OF WOLBACHIA INTO AEDES AEGYPTI MOSQUITOES TO REDUCE DENGUE TRANSMISSION
}

\author{
MEKSIANIS Z. NDII ${ }^{\otimes 1,2,3}$, ROSLYN I. HICKSON ${ }^{3,4}$ and GEOFFRY N. MERCER ${ }^{4}$
}

(Received 13 February, 2012; revised 13 August, 2012)

\begin{abstract}
Infecting Aedes aegypti mosquitoes with the bacteria Wolbachia has been proposed as an innovative new strategy to reduce the transmission of dengue fever. Field trials are currently being undertaken in Queensland, Australia. However, few mathematical models have been developed to consider the persistence of Wolbachiainfected mosquitoes in the wild. This paper develops a mathematical model to determine the persistence of Wolbachia-infected mosquitoes by considering the competition between Wolbachia-infected and non-Wolbachia mosquitoes. The model has four steady states that are biologically feasible: all mosquitoes dying out, only non-Wolbachia mosquitoes surviving, and two steady states where non-Wolbachia and Wolbachiainfected mosquitoes coexist. The stability of the steady states is determined with respect to the key parameters in the mosquito life cycle. A global sensitivity analysis of the model is also conducted. The results show that the persistence of Wolbachia-infected mosquitoes is dominated by the reproductive rate, death rate, maturation rate and maternal transmission. For the parameter values where Wolbachia persists, it dominates the population, and hence the introduction of Wolbachia has great potential to reduce dengue transmission.
\end{abstract}

2010 Mathematics subject classification: primary 92D25; secondary 93D30.

Keywords and phrases: Wolbachia, Aedes aegypti, dengue, mathematical model, sensitivity analysis, stability.

\section{Introduction}

Dengue, a vector-borne disease, is a public health problem worldwide and poses a risk to two-thirds of the world's population [1]. There are four distinct serotype viruses of dengue: DENV1, DENV2, DENV3 and DENV4. Infection with dengue is divided

\footnotetext{
${ }^{1}$ Mathematical Sciences Institute, The Australian National University, Canberra, ACT 0200, Australia.

${ }^{2}$ Department of Mathematics, The University of Nusa Cendana, East Nusa Tenggara, Indonesia; e-mail: meksand@gmail.com.

${ }^{3}$ School of Mathematical and Physical Sciences, The University of Newcastle, Callaghan, NSW 2308, Australia; e-mail: R.Hickson@UNSWalumni.com.

${ }^{4}$ National Centre for Epidemiology and Population Health, The Australian National University, Canberra, ACT 0200, Australia; e-mail: Geoff.Mercer@anu.edu.au.

(C) Australian Mathematical Society 2012, Serial-fee code 1446-1811/2012\$16.00
} 
into three categories: dengue fever, dengue haemorrhagic fever (DHF), and dengue shock syndrome (DSS). DHF and DSS are the most severe forms of dengue, with fatality rates greater than $20 \%$ [17]. If individuals are infected by one serotype of the virus, they become immune to that serotype. However, they are more likely to contract the much more severe DHF and DSS if they are subsequently infected with any of the other three serotypes.

Aedes aegypti is the mosquito that is responsible for most dengue transmission globally. Its lifespan is 10-22 days, depending on the temperature and humidity of the region [19]. It is common in urban areas, has a preference for laying eggs in small artificial water sources such as pot plant holders, water tanks, and discarded tyres [10], and is thought to move approximately $120 \mathrm{~m}$ during its lifetime [9]. It is highly anthropophilic, preferring to feed on humans over animals.

Various strategies have been implemented to reduce dengue transmission by controlling the mosquito vector, but these have been largely ineffective. For example, the use of insecticide has become largely ineffective as the mosquitoes have developed resistance to the chemicals [8]. Environmental measures such as house-to-house removal of breeding sites are effective in the short term but are expensive and require continuous application.

An alternative approach of infecting Aedes aegypti mosquitoes with Wolbachia has been proposed as a new strategy for reducing dengue transmission [7]. Wolbachia is a bacterium that can inhibit the ability of Aedes aegypti to transmit dengue in different ways depending on its strain. One strain of Wolbachia achieves this by shortening the lifespan of the mosquitoes, resulting in the dengue virus not having sufficient time to replicate in the mosquito to a level where it is infectious [3]. This strain bends the proboscis [15], so that it is unable to pierce the skin. Another strain of Wolbachia reduces how much dengue virus is present in the saliva of the mosquito [16]. Experimental results and current field trials show that the ability of Aedes aegypti mosquitoes infected with Wolbachia to transmit dengue is reduced $[2,7,16]$.

On its own, the reduction in the mosquito lifespan would result in Wolbachiainfected mosquitoes being unable to compete in the wild with non-Wolbachia mosquitoes. That is, the Wolbachia-infected mosquitoes would not persist. However, Wolbachia gives female mosquitoes a reproductive advantage known as "cytoplasmic incompatibility" (CI). CI is the condition where Wolbachia causes incompatibility between Wolbachia-infected males and non-Wolbachia females, such that a cross between the two produces embryos that die before hatching. To summarize the effect of CI, there are four possibilities to consider:

(1) Non-Wolbachia males and non-Wolbachia females produce uninfected offspring.

(2) Non-Wolbachia males and Wolbachia-infected females produce both Wolbachiainfected and uninfected offspring in a certain ratio.

(3) Wolbachia-infected males and non-Wolbachia females cannot produce offspring successfully (although an embryo is formed), further blocking reproduction of non-Wolbachia females. 
(4) Wolbachia-infected males and Wolbachia-infected females produce both infected and uninfected offspring in a certain ratio.

There is, therefore, a complex interplay between lifespan shortening and CI that gives rise to potentially complex dynamics in the mosquito population. If the Wolbachiainfected mosquitoes can dominate the population, a reduction in dengue transmission can be attained.

To date, few mathematical models have been developed to determine the persistence of Wolbachia-infected Aedes aegypti mosquitoes. Previous models have not considered the competition for persistence between non-Wolbachia and Wolbachiainfected mosquitoes [16]. Therefore, in this paper, a deterministic model for a mosquito population including the introduction of Wolbachia-infected mosquitoes into the wild is developed and analysed.

This paper is organized as follows. The model is developed and the key mosquito life-cycle parameters are explained in Section 2. The steady states of the model are found and their stability is determined in Section 3. Numerical solutions are calculated and discussed in Section 4. A sensitivity analysis is conducted in Section 5 to determine which parameters dominate the mosquito population dynamics. Finally, conclusions are presented in Section 6.

\section{Model development}

The model is a deterministic compartment model with the mosquito population represented by six compartments, as shown in Figure 1. The compartments represent non-Wolbachia aquatic mosquitoes $\left(A_{N}\right)$, non-Wolbachia male and female mosquitoes $\left(M_{N}\right.$ and $F_{N}$, respectively), Wolbachia-infected aquatic mosquitoes $\left(A_{W}\right)$, and Wolbachia-infected male and female mosquitoes ( $M_{W}$ and $F_{W}$, respectively). The total adult population is the sum of non-Wolbachia and Wolbachia-infected adult male and female mosquitoes, $P=M_{N}+F_{N}+M_{W}+F_{W}$. The model assumes homogeneous mixing and does not capture the dispersal of mosquitoes. As the model aims to investigate whether Wolbachia-infected mosquitoes can persist in competition with non-Wolbachia mosquitoes, these assumptions do not have an impact on the model outcomes. Eggs, larvae and pupae are grouped into one aquatic stage instead of dividing them into different compartments since Wolbachia does not influence the immature stage. The adult stage is divided into two compartments, male and female, to enable the inclusion of $\mathrm{CI}$ in reproduction.

The lines in Figure 1 show how mosquitoes progress through the system as they mature. Non-Wolbachia aquatic mosquitoes only become non-Wolbachia adults. Maternal transmission of Wolbachia is not $100 \%$, hence Wolbachia-infected aquatic mosquitoes can mature to become either Wolbachia-infected adults with the proportion $\alpha_{W}$ or non-Wolbachia adults with the proportion $1-\alpha_{W}$. The unlabelled arrows into $A_{N}$ and $A_{W}$ represent the reproductive rate, indicating birth, which is influenced by the size of the male and female adult populations. 


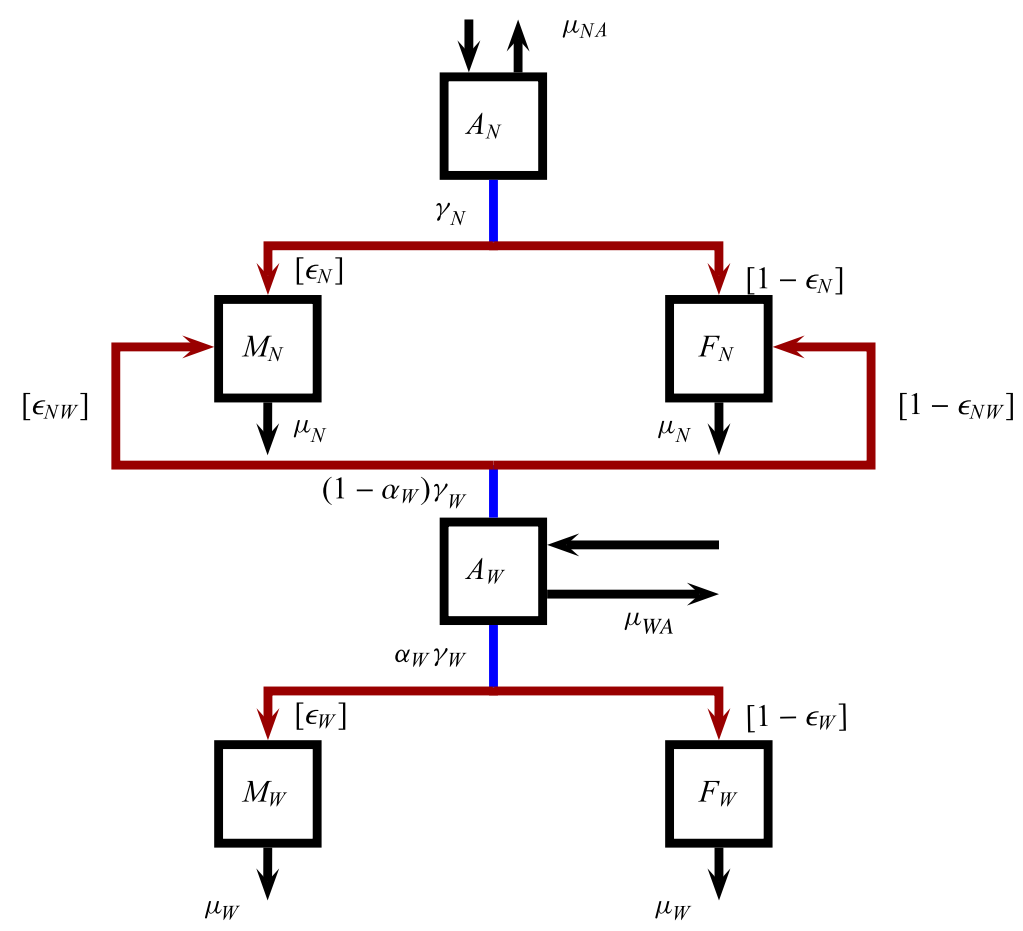

FIGURE 1. Schematic representation of the model (2.1). Unbracketed labels are rates and bracketed labels are proportions. The unlabelled arrows into $A_{N}$ and $A_{W}$ represent the reproductive rate, indicating birth, which is influenced by the size of the male and female adult populations.

The mosquito population model is governed by the following system of differential equations:

$$
\begin{aligned}
\frac{d A_{N}}{d t} & =\rho_{N} \frac{F_{N} M_{N}}{P}\left(1-\frac{A_{N}+A_{W}}{K}\right)-\mu_{N A} A_{N}-\gamma_{N} A_{N}, \\
\frac{d M_{N}}{d t} & =\epsilon_{N} \gamma_{N} A_{N}-\mu_{N} M_{N}+\epsilon_{N W}\left(1-\alpha_{W}\right) \gamma_{W} A_{W}, \\
\frac{d F_{N}}{d t} & =\left(1-\epsilon_{N}\right) \gamma_{N} A_{N}-\mu_{N} F_{N}+\left(1-\epsilon_{N W}\right)\left(1-\alpha_{W}\right) \gamma_{W} A_{W}, \\
\frac{d A_{W}}{d t} & =\rho_{W} \frac{F_{W}\left(M_{W}+M_{N}\right)}{P}\left(1-\frac{A_{N}+A_{W}}{K}\right)-\mu_{W A} A_{W}-\gamma_{W} A_{W}, \\
\frac{d M_{W}}{d t} & =\epsilon_{W} \alpha_{W} \gamma_{W} A_{W}-\mu_{W} M_{W}, \\
\frac{d F_{W}}{d t} & =\left(1-\epsilon_{W}\right) \alpha_{W} \gamma_{W} A_{W}-\mu_{W} F_{W} .
\end{aligned}
$$

An increase in non-Wolbachia aquatic mosquitoes $\left(A_{N}\right)$ is determined by the reproductive rate $\rho_{N}$ and occurs when non-Wolbachia female and male mosquitoes 
mate and produce offspring. A decrease in non-Wolbachia aquatic mosquitoes occurs due to maturation of non-Wolbachia aquatic mosquitoes at rate $\gamma_{N}$ and their death at rate $\mu_{N A}$. The number of Wolbachia-infected aquatic mosquitoes $\left(A_{W}\right)$ increases as Wolbachia-infected females $\left(F_{W}\right)$ mate with non-Wolbachia and Wolbachia-infected males $\left(M_{N}\right.$ and $\left.M_{W}\right)$ and produce offspring at rate $\rho_{W}$. This number decreases due to death at rate $\mu_{W A}$ and maturation at rate $\gamma_{W}$.

The number of non-Wolbachia adult mosquitoes increases as non-Wolbachia aquatic mosquitoes mature at rate $\gamma_{N}$ and some proportion $\epsilon_{N}$ become male and the rest $\left(1-\epsilon_{N}\right)$ become female. This is similar for Wolbachia-infected adults. In addition, a proportion $\alpha_{W}$ of infected aquatic mosquitoes mature to become Wolbachia-infected adults and a proportion $1-\alpha_{W}$ mature to become non-Wolbachia adults. The death rates of Wolbachia-infected and non-Wolbachia adults are denoted by $\mu_{W}$ and $\mu_{N}$, respectively.

The effect of CI is evident in the equations for the aquatic stages, with the difference being the $F_{N} M_{N} / P$ term for non-Wolbachia aquatics compared to $F_{W}\left(M_{W}+M_{N}\right) / P$ for Wolbachia-infected aquatics. The parameter $K$ is the carrying capacity, which is the capacity of the environment to sustain the aquatic mosquitoes. The populations can be nondimensionalized with respect to this value, which is equivalent to setting $K=1$.

\section{Steady states and stability}

This section presents the steady states of the model (2.1) and their stability. As the ratio between male and female mosquitoes is approximately 1:1 [12], and letting $K=1$, (2.1) can be reduced to

$$
\begin{aligned}
\frac{d A_{N}}{d t} & =\rho_{N} \frac{M_{N}^{2}}{2\left(M_{N}+M_{W}\right)}\left[1-\left(A_{N}+A_{W}\right)\right]-\mu_{N A} A_{N}-\gamma_{N} A_{N}, \\
\frac{d M_{N}}{d t} & =\frac{\gamma_{N} A_{N}}{2}-\mu_{N} M_{N}+\frac{\left(1-\alpha_{W}\right) \gamma_{W} A_{W}}{2}, \\
\frac{d A_{W}}{d t} & =\frac{\rho_{W} M_{W}}{2}\left[1-\left(A_{N}+A_{W}\right)\right]-\mu_{W A} A_{W}-\gamma_{W} A_{W}, \\
\frac{d M_{W}}{d t} & =\frac{\alpha_{W} \gamma_{W} A_{W}}{2}-\mu_{W} M_{W} .
\end{aligned}
$$

The model has four steady-state solutions:

$$
E_{1}=(0,0,0,0), \quad E_{2}=\left(A_{N}^{*}, M_{N}^{*}, 0,0\right), \quad E_{3}, E_{4}=\left(A_{N}^{+}, M_{N}^{+}, A_{W}^{+}, M_{W}^{+}\right),
$$

where

$$
\begin{aligned}
& A_{N}^{*}=1-\frac{4 \mu_{N}\left(\mu_{N A}+\gamma_{N}\right)}{\rho_{N} \gamma_{N}}, \\
& M_{N}^{*}=\frac{\gamma_{N} A_{N}^{*}}{2 \mu_{N}}=\frac{\gamma_{N}}{2 \mu_{N}}\left(1-\frac{4 \mu_{N}\left(\mu_{N A}+\gamma_{N}\right)}{\rho_{N} \gamma_{N}}\right),
\end{aligned}
$$


and

$$
M_{N}^{+}=\frac{1}{2 \mu_{N}}\left(B_{4} A_{N}^{+}+B_{5}\right), \quad M_{W}^{+}=B_{3} B_{2}-B_{3} A_{N}^{+}, \quad A_{W}^{+}=B_{2}-A_{N}^{+},
$$

with $A_{N}^{+}$given by the nonnegative roots of $K_{1} A_{N}^{+2}+K_{2} A_{N}^{+}+K_{3}=0$, where

$$
\begin{aligned}
K_{1} & =\left(\frac{1}{B_{1}}-\frac{B_{4}}{2 \mu_{N}}\right) \frac{B_{4}}{2 \mu_{N}}-\frac{B_{3}}{B_{1}}, \\
K_{2} & =\left(\frac{1}{B_{1}}-\frac{B_{4}}{2 \mu_{N}}\right) \frac{B_{5}}{2 \mu_{N}}+\frac{B_{3} B_{2}}{B_{1}}-\frac{B_{4} B_{5}}{4 \mu_{N}^{2}}, \quad K_{3}=-\frac{B_{5}^{2}}{4 \mu_{N}^{2}},
\end{aligned}
$$

where $B_{1}, \ldots, B_{5}$ are defined in terms of the parameter values as

$$
\begin{aligned}
& B_{1}=\frac{2 \rho_{N}}{\mu_{N A}+\gamma_{N}} \frac{\mu_{W}\left(\mu_{W A}+\gamma_{W}\right)}{\rho_{W} \gamma_{W} \alpha_{W}}, \quad B_{2}=1-\frac{4 \mu_{W}\left(\mu_{W A}+\gamma_{W}\right)}{\rho_{W} \gamma_{W} \alpha_{W}}, \\
& B_{3}=\frac{\gamma_{W} \alpha_{W}}{2 \mu_{W}}, \quad B_{4}=\gamma_{N}-\left(1-\alpha_{W}\right) \gamma_{W}, \quad B_{5}=\left(1-\alpha_{W}\right) \gamma_{W} B_{2} .
\end{aligned}
$$

As $A_{N}^{+}$is calculated from a quadratic equation, there are two possible values, resulting in two possible steady states $\left(E_{3}\right.$ and $\left.E_{4}\right)$. Whether $A_{N}^{+}$is physically realistic or not depends on the parameter values.

The steady state $E_{1}$, where all mosquitoes die out, is not an interesting case as this does not occur in reality. The steady state $E_{2}$ is where non-Wolbachia mosquitoes persist and Wolbachia-infected mosquitoes die out. This is one of the steady states of interest as it means that non-Wolbachia mosquitoes dominate the population and hence transmission of dengue continues at the unreduced level. The steady states $E_{3}$ and $E_{4}$ are when both non-Wolbachia and Wolbachia-infected mosquitoes persist. It is required that the steady states be physically realistic, which implies that they should be nonnegative as they represent a proportion of the carrying capacity. There is a special case if perfect maternal transmission $\alpha_{W}=1$ occurs. That is, only Wolbachia-infected mosquitoes persist $\left(A_{N}=M_{N}=0\right)$. When maternal transmission is perfect $\left(\alpha_{W}=1\right)$, $B_{5}=0$ and $K_{3}=0$ and hence $A_{N}^{+}=0$ is the only realistic solution. However, this is not biologically realistic as in reality perfect maternal transmission cannot happen [16].

The stability of the steady states is determined by calculating the Jacobian matrix and finding the eigenvalues. If the real parts of all the eigenvalues are negative, then the steady state is locally stable; otherwise, it is unstable. For biologically sensible parameter values, the steady state where all mosquitoes die out $\left(E_{1}\right)$ was found to be unstable. The steady state where Wolbachia-infected mosquitoes die out and non-Wolbachia mosquitoes persist $\left(E_{2}\right)$ was found to be stable for a range of biologically sensible parameter values. As the Wolbachia-infected mosquitoes die out, the model is reduced to a simple mosquito population model without Wolbachiainfected mosquitoes. It is straightforward to show that $E_{2}$ is stable if

$$
\rho_{N}>\frac{\left(\mu_{N A}+\gamma_{N}\right) \mu_{N}}{\gamma_{N} \epsilon_{N}\left(1-\epsilon_{N}\right)}
$$




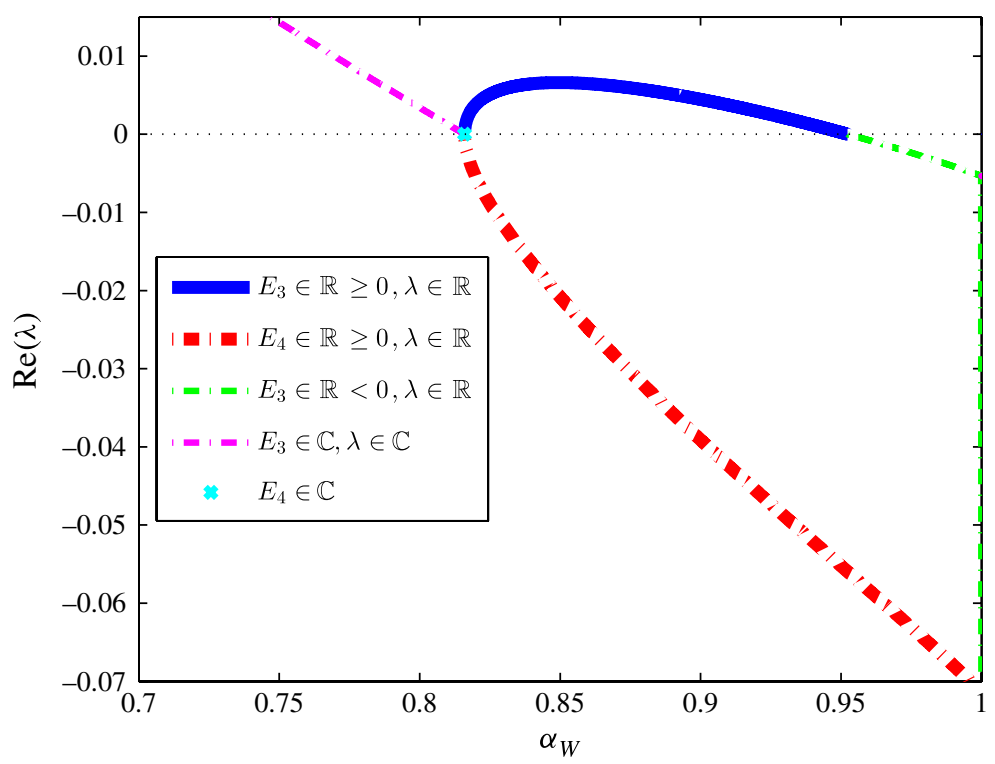

FIgurE 2. Stability of steady states $E_{3}$ and $E_{4}$. The largest real parts of the eigenvalues are plotted against the maternal transmission $\alpha_{W}$, with $\rho_{N}=1.25, \rho_{W}=2.1 \rho_{N}$ and the other parameter values given by Table 1 . A steady state must be real and positive to be physically realistic. (Colour available online.)

The stability of the two steady states where both non-Wolbachia and Wolbachiainfected mosquitoes persist $\left(E_{3}\right.$ and $\left.E_{4}\right)$ is obtained numerically. Figure 2 shows the stability of the surviving steady states by plotting the largest real part of the eigenvalues (denoted by $\lambda$ ) against the maternal transmission parameter values $\left(\alpha_{W}\right)$. The thick lines are physically realistic steady states and the thin lines are unrealistic (negative). When the steady states are real and positive, hence realistic, the eigenvalues can be real or complex. The stability of the steady states is determined by the sign of the real part of the eigenvalues: if it is negative, the steady state is stable; otherwise, it is unstable.

There are two realistic steady states. As shown by the sign of the largest real part of the eigenvalues, only one of them is stable. For $\alpha_{W} \lesssim 0.816$, the steady state $E_{3}$ is real but negative, and further analysis shows that in this case the Wolbachia-free steady state $E_{2}$ is stable. This is biologically sensible as for low maternal transmission $\left(\alpha_{W}\right)$, the number of Wolbachia-infected offspring produced is low and hence the number of non-Wolbachia mosquitoes is high. Therefore, Wolbachia-infected mosquitoes die out. Furthermore, when $0.816 \lesssim \alpha_{W}<1$, the $E_{4}$ steady state is real, positive and stable, whereas $E_{3}$ is either real, positive and unstable, or negative (physically unrealistic) but stable. Therefore, there is only one physically realistic stable steady state where both non-Wolbachia and Wolbachia-infected mosquitoes coexist. A similar approach can be implemented to determine the stability of the steady states in terms of the other parameters. 
TABLE 1. Parameter descriptions, values and sources for the model (2.1) for introducing Wolbachiainfected mosquitoes into the wild.

\begin{tabular}{|c|c|c|c|c|}
\hline Parameter & Description & Value & Unit & Source \\
\hline$\rho_{N}$ & Non-Wolbachia reproductive rate & 1.25 & day $^{-1}$ & Estimated \\
\hline$\rho_{W}$ & Wolbachia reproductive rate & $2.1 \rho_{N}$ & $\mathrm{day}^{-1}$ & Estimated \\
\hline$\mu_{N A}$ & Non-Wolbachia aquatic death rate & $1 / 7.78$ & day $^{-1}$ & [19] \\
\hline $\begin{array}{l}\mu_{N A} \\
\mu_{W A}\end{array}$ & Wolbachia aquatic death rate & $1 / 7.78$ & $\mathrm{day}^{-1}$ & {$[2,16,19]$} \\
\hline$\gamma_{N}$ & Non-Wolbachia maturation rate & $1 / 6.67$ & day $^{-1}$ & {$[5,19]$} \\
\hline$\gamma_{W}$ & Wolbachia maturation rate & $1 / 6.67$ & day $^{-1}$ & {$[2,5,16]$} \\
\hline$\epsilon_{N}$ & $\begin{array}{l}\text { Proportion of non-Wolbachia } \\
\text { adults that are male }\end{array}$ & 0.5 & N/A & {$[12,19]$} \\
\hline$\epsilon_{W}$ & $\begin{array}{l}\text { Proportion of Wolbachia-infected } \\
\text { adults that are male }\end{array}$ & 0.5 & N/A & {$[2]$} \\
\hline$\mu_{N}$ & Non-Wolbachia adult death rate & $1 / 14$ & $\mathrm{day}^{-1}$ & [4] \\
\hline$\mu_{W}$ & Wolbachia adult death rate & $1 / 7$ & $\mathrm{day}^{-1}$ & {$[11,16]$} \\
\hline$\epsilon_{N W}$ & $\begin{array}{l}\text { Proportion of non-Wolbachia males } \\
\text { hatched from a Wolbachia-infected mother }\end{array}$ & 0.5 & $\mathrm{~N} / \mathrm{A}$ & [12] \\
\hline$\alpha_{W}$ & Maternal transmission & $0.8-0.9$ & $\mathrm{~N} / \mathrm{A}$ & {$[13,16,18,20]$} \\
\hline$P$ & Total population & $\begin{array}{l}M_{N}+M_{W} \\
\quad+F_{N}+F_{W}\end{array}$ & N/A & N/A \\
\hline K & Carrying capacity & 1 & $\mathrm{~N} / \mathrm{A}$ & N/A \\
\hline
\end{tabular}

\section{Numerical solutions}

Numerical solutions of the model (2.1) are calculated using the routine ODE15s in MATLAB. The parameter values are taken from the available literature and summarized in Table 1 . As no data is currently available to the authors, validation and calibration of the model against data will be conducted as it becomes available. Initial values for the numerical simulations are obtained from calculation of the steady state of the model without the introduction of Wolbachia-infected mosquitoes. The initial population of Wolbachia-infected mosquitoes $\left(A_{W}+M_{W}+F_{W}\right)$ is $20 \%$ of the total population of non-Wolbachia mosquitoes. This was done to replicate the experiments of Xi et al. [18]. The rate $\rho_{W}$ is estimated to be higher than $\rho_{N}$ because Wolbachiainfected mosquitoes have higher fecundity rates [12]. The estimated value of the non-Wolbachia reproductive rate, $\rho_{N}=1.25$, is used because this value in combination with other parameter values ensures that non-Wolbachia mosquitoes persist in the wild before the introduction of Wolbachia-infected mosquitoes, so as to reflect reality.

Figure 3 shows a case where Wolbachia-infected mosquitoes die out and nonWolbachia mosquitoes persist, using $\alpha_{W}=0.8$. Of interest in terms of the experimental results and seasonal factors is how long this takes. For this example, it takes approximately 300 days for the Wolbachia-infected mosquitoes to die out. Figure 3 shows that the number of non-Wolbachia mosquitoes decreases in the early period due to competition with the added Wolbachia-infected mosquitoes, before increasing and approaching the steady state. 


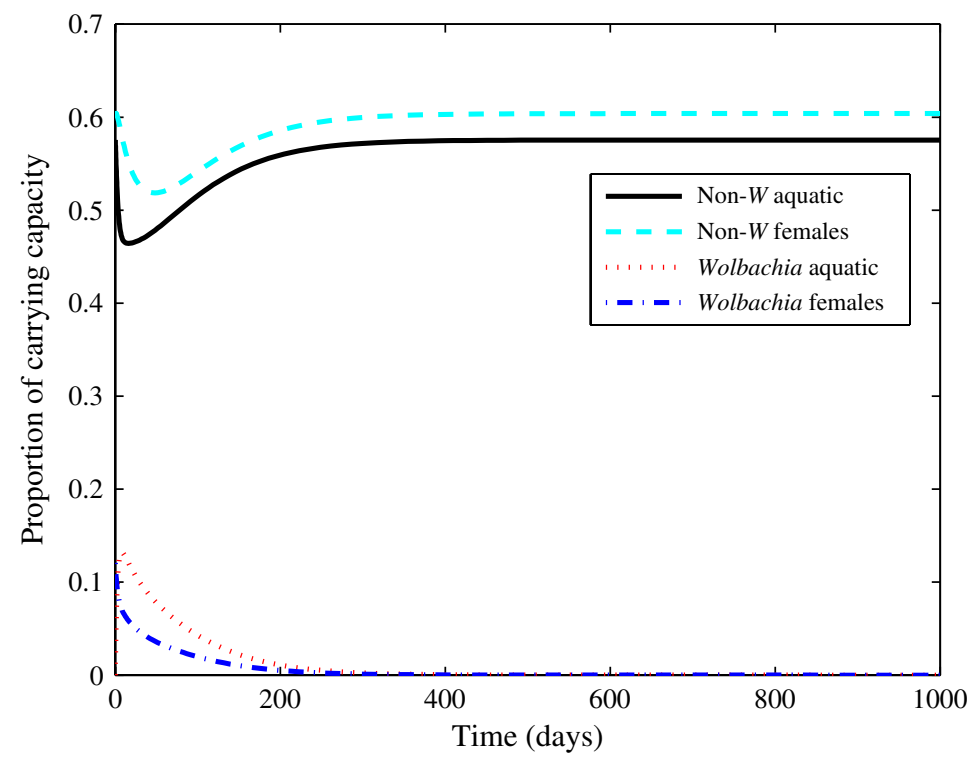

FIGURE 3. Numerical solution where the Wolbachia-infected mosquitoes die out, with $\alpha_{W}=0.8$ and the other parameter values given by Table 1 . The initial Wolbachia-infected population is $20 \%$ of the total population of non-Wolbachia mosquitoes.

Figure 4 shows a case where persistence of both non-Wolbachia mosquitoes and Wolbachia-infected mosquitoes occurs, using $\alpha_{W}=0.9$. It takes approximately 450 days for the mosquito population to approach this steady state. The number of Wolbachia-infected female mosquitoes declines slightly in the early period due to competition for resources, but after that they increase and dominate the population.

In comparing Figures 3 and 4, the only change in the parameter values is that the maternal transmission has been increased from $\alpha_{W}=0.8$ in Figure 3 to $\alpha_{W}=$ 0.9 in Figure 4. The result confirms the stability analysis of the steady state as given in Figure 2, where the Wolbachia-infected mosquitoes die out if $\alpha_{W} \lesssim$ 0.816, and otherwise both non-Wolbachia and Wolbachia-infected mosquitoes persist. Furthermore, this result indicates the sensitivity of the model to the parameter, where a slight change of the parameter value can lead to a different model outcome. Further investigation of this is conducted in Section 5.

Experimental results conducted in far north Queensland for approximately 90-120 days [16] show that Wolbachia-infected mosquitoes persist. Our numerical results show that Wolbachia-infected mosquitoes persist longer than approximately 120 days, but over a longer period the population dies out (see Figure 3). Furthermore, when the maternal transmission is high, $\alpha_{W}=0.9$, Wolbachia-infected mosquitoes persist, but it takes around 450 days to approach the steady state. This is longer than the 120 days in the aforementioned experiments [16], meaning that the steady state shown here is not likely to be reached in far north Queensland given seasonality. Therefore, our results 


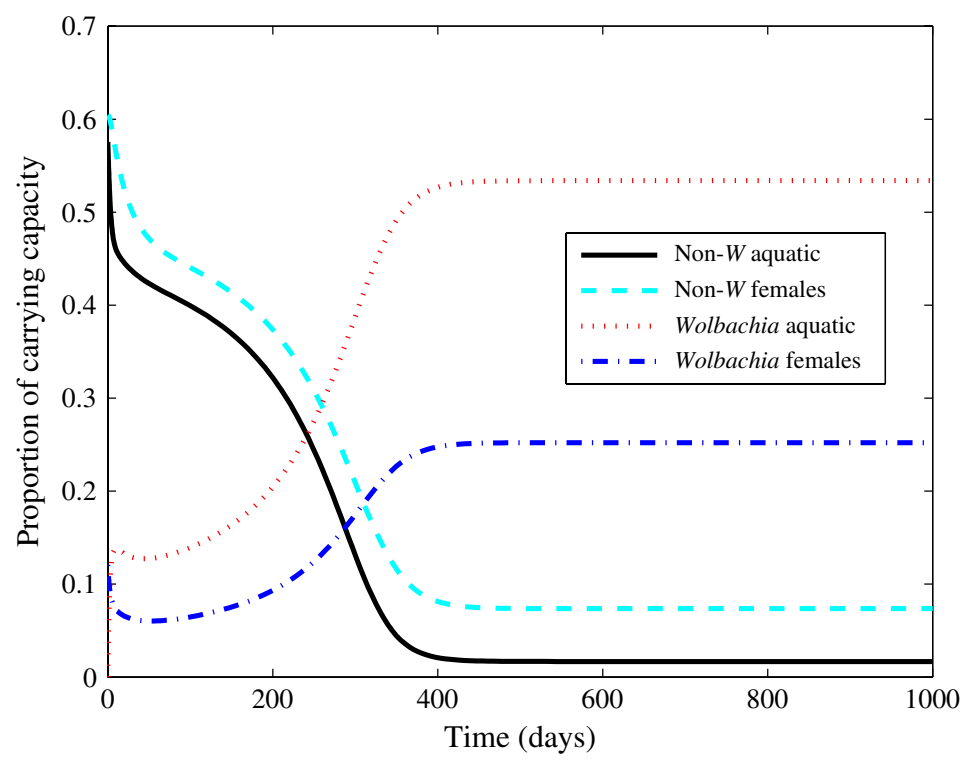

FIGURE 4. Numerical solution where the Wolbachia-infected mosquitoes persist, with $\alpha_{W}=0.9$ and the other parameter values given by Table 1 . The initial Wolbachia-infected population is $20 \%$ of the total population of non-Wolbachia mosquitoes.

reveal that there is a possibility for Wolbachia-infected mosquitoes to die out in the long term, something that cannot be determined by experimental trials conducted for a relatively short period of approximately 90-120 days.

The persistence of Wolbachia-infected mosquitoes is possible, as in the experimental trials, weekly supplementation of Wolbachia-infected mosquitoes was undertaken $[2,16]$. However, the experimental trials did not present the number of non-Wolbachia mosquitoes, only showing that Wolbachia-infected mosquitoes persist. It may happen that the number of Wolbachia-infected mosquitoes is smaller than the number of non-Wolbachia mosquitoes. This means that non-Wolbachia mosquitoes still dominate the population, and hence the transmission of dengue continues. The numerical results presented in this paper clearly show the numbers of non-Wolbachia and Wolbachia-infected mosquitoes when competing in the wild.

\section{Sensitivity analysis}

Sensitivity of the model outcome to the parameters is investigated in this section. Latin hypercube sampling is used in conjunction with partial rank correlation coefficient (PRCC) multivariate analysis [6] to investigate which parameters most contribute to the model outcome. The parameters are sampled from a triangular probability distribution since a most likely value (peak), a minimum and a maximum value for each parameter are estimable [14]. A negative PRCC indicates a negative correlation between parameters and the outcome of interest. This means that an 
increase in the parameter value leads to a decrease in the outcome of interest. On the other hand, a positive PRCC indicates a positive correlation between parameters and the outcome of interest, meaning that an increase in the parameter value leads to an increase in the outcome of interest. The larger the magnitude of the PRCC value, the more dominant that parameter is in the outcome.

The parameters are chosen in such a way that for one group of parameter values, the steady state is usually the Wolbachia-free steady state, which is where Wolbachiainfected mosquitoes die out and non-Wolbachia mosquitoes persist $\left(E_{2}\right)$. For the other group of parameter values, the steady state is where both Wolbachia-infected and non-Wolbachia mosquitoes persist $\left(E_{4}\right)$. The sensitivity of the model outcome to the parameters is measured against a monotonically increasing function. Of interest is the cumulative number of female mosquitoes, since they are responsible for dengue transmission and hence are a proxy for dengue activity. For the range of parameters where the model outcome tends to the Wolbachia-free steady state $\left(E_{2}\right)$, the parameters are measured against the cumulative number of non-Wolbachia female mosquitoes, which is the solution of

$$
\frac{d C_{F N}}{d t}=\left(1-\epsilon_{N}\right) \gamma_{N} A_{N}+\epsilon_{N W}\left(1-\alpha_{W}\right) \gamma_{W} A_{W}
$$

For the range of parameters where the model outcome tends to the Wolbachiasurviving steady state $\left(E_{4}\right)$, the parameters are measured against both the cumulative number of non-Wolbachia female mosquitoes (the solution of (5.1)) and the cumulative number of Wolbachia-infected female mosquitoes, which is the solution of

$$
\frac{d C_{F W}}{d t}=\left(1-\epsilon_{W}\right) \alpha_{W} \gamma_{W} A_{W} .
$$

Over 2000 runs of the model are performed, with at least $95 \%$ of the runs tending to one of the steady states of interest. Only samples resulting in the correct steady state are kept for each PRCC analysis, resulting in at least 2000 samples. The range of parameter values used for each steady-state analysis is given in Table 2 . In addition, $\rho_{N}$ and $\rho_{W}$ are not correlated. They are sampled randomly from the defined range of parameter values. Note that only $\mu_{W}$ and $\rho_{W}$ are changed to alternate between $E_{2}$ and $E_{4}$.

As summarized in Table 3, when Wolbachia-infected mosquitoes die out and nonWolbachia mosquitoes persist $\left(E_{2}\right)$, non-Wolbachia related parameters are dominant (larger magnitude) when the parameters are measured against the cumulative number of non-Wolbachia female mosquitoes. This is biologically sensible, as only nonWolbachia mosquitoes persist. The parameters $\mu_{N A}$ and $\mu_{N}$ have negative PRCC values, meaning that an increase in the death rates of non-Wolbachia aquatics and nonWolbachia adults reduces the population of non-Wolbachia mosquitoes. On the other hand, the parameters $\rho_{N}$ and $\gamma_{N}$ have positive PRCC values, indicating that as the reproductive rate or the maturation rate of non-Wolbachia mosquitoes increases, the population increases. In contrast, when both non-Wolbachia and Wolbachia-infected 
TABle 2. Parameter descriptions, minimum, mode and maximum values and references. Where two ranges of parameters are given, the first is for the Wolbachia-free steady state $\left(E_{2}\right)$, and the second is for the steady state where Wolbachia-infected mosquitoes survive $\left(E_{4}\right)$.

\begin{tabular}{llllll}
\hline Parameter & Min & Mode & Max & Unit & Source \\
\hline$\rho_{N}$ & 1 & 1.25 & 2.5 & $\mathrm{day}^{-1}$ & Estimated \\
$\mu_{N A}$ & $1 / 16.84$ & $1 / 7.78$ & $1 / 3.5$ & $\mathrm{day}^{-1}$ & {$[19]$} \\
$\gamma_{N}$ & $1 / 13.51$ & $1 / 6.67$ & $1 / 5$ & $\mathrm{day}^{-1}$ & {$[5,19]$} \\
$\mu_{N}$ & $1 / 22.77$ & $1 / 14$ & $1 / 10$ & $\mathrm{day}^{-1}$ & {$[12,19]$} \\
$\mu_{W A}$ & $1 / 16.84$ & $1 / 7.78$ & $1 / 3.5$ & $\mathrm{day}^{-1}$ & {$[2,16,19]$} \\
$\mu_{W}$ & $1 / 11$ & $1 / 7$ & $1 / 5$ & $\mathrm{day}^{-1}$ & {$[11,16]$} \\
& $1 / 20$ & $1 / 12$ & $1 / 8$ & & \\
$\rho_{W}$ & $\rho_{N}$ & $1.25 \rho_{N}$ & $1.5 \rho_{N}$ & $\mathrm{day}^{-1}$ & Estimated \\
& $2 \rho_{N}$ & $2.5 \rho_{N}$ & $6 \rho_{N}$ & & \\
$\gamma_{W}$ & $1 / 13.51$ & $1 / 6.67$ & $1 / 5$ & $\mathrm{day}^{-1}$ & {$[2,5,16]$} \\
$\alpha_{W}$ & 0.8 & 0.9 & 1 & $\mathrm{~N} / \mathrm{A}$ & {$[13,16,18,20]$} \\
\hline
\end{tabular}

TABLE 3. PRCC values for the Wolbachia-free steady state $\left(E_{2}\right)$ and for the both-surviving steady state $\left(E_{4}\right)$ after 1000 days, using parameter ranges given in Table 2. Here $C_{F N}$ is the cumulative number of non-Wolbachia female mosquitoes from equation (5.1), and $C_{F W}$ is the cumulative number of Wolbachiainfected female mosquitoes from equation (5.2).

\begin{tabular}{lrrrr}
\hline \multirow{2}{*}{ Parameter } & \multicolumn{1}{c}{$E_{2}$} & & \multicolumn{2}{c}{$E_{4}$} \\
\cline { 2 - 3 } \cline { 5 - 5 } & $C_{F N}$ & & $C_{F N}$ & $C_{F W}$ \\
\hline$\rho_{N}$ & 0.8854 & & 0.4279 & -0.0158 \\
$\mu_{N A}$ & -0.8303 & & -0.3523 & 0.1575 \\
$\gamma_{N}$ & 0.9644 & & 0.2952 & -0.0150 \\
$\mu_{N}$ & -0.8297 & & -0.4206 & 0.1454 \\
$\mu_{W A}$ & -0.0051 & & 0.0133 & -0.6142 \\
$\mu_{W}$ & 0.0708 & & 0.0132 & -0.6902 \\
$\rho_{W}$ & 0.0071 & & 0.0736 & 0.8000 \\
$\gamma_{W}$ & 0.0053 & & 0.7499 & 0.9717 \\
$\alpha_{W}$ & -0.0225 & & -0.9499 & 0.7790 \\
\hline
\end{tabular}

mosquitoes persist $\left(E_{4}\right)$, Wolbachia-infected related parameters are dominant. For example, when measured against the cumulative number of non-Wolbachia female mosquitoes $\left(C_{F N}\right)$, the parameters $\alpha_{W}$ and $\gamma_{W}$ have the highest magnitudes. This means that if the maternal transmission rate or the maturation rate increases, the population of Wolbachia-infected mosquitoes increases. The Wolbachia-related parameters are also dominant when measured against the cumulative number of Wolbachia-infected mosquitoes $\left(C_{F W}\right)$.

The results of the sensitivity analysis reveal that once the Wolbachia-infected mosquitoes persist, they dominate the mosquito population. This confirms that the introduction of Wolbachia-infected mosquitoes can be a powerful strategy in reducing dengue transmission. Furthermore, a greater reduction in non-Wolbachia female 
mosquitoes can be obtained if the proportion of Wolbachia-infected offspring from a Wolbachia-infected mother $\left(\alpha_{W}\right)$ is high, as given by the PRCC value of $\alpha_{W}$ $(-0.9499)$ when measured against the cumulative number of non-Wolbachia female mosquitoes.

\section{Conclusions}

Research conducted by Hoffman et al. [2] and Walker et al. [16] found that Wolbachia-infected mosquitoes will dominate the population. Hence a Wolbachia intervention would be a potential strategy to reduce dengue spread. These findings are compatible with what the proposed model has demonstrated. The numerical solutions, stability analysis and sensitivity analysis show that once the Wolbachiainfected mosquitoes persist, they can ultimately dominate the population. The model also reveals that the persistence of Wolbachia-infected mosquitoes mostly depends on the death rate of Wolbachia-infected adults $\left(\mu_{W}\right)$, the maturation rate of Wolbachia-infected adults $\left(\gamma_{W}\right)$, their reproductive rate $\left(\rho_{W}\right)$ and maternal transmission $\left(\alpha_{W}\right)$. The non-Wolbachia mosquitoes would dominate the population only if the Wolbachia-infected mosquitoes die out. This means that once the Wolbachia-infected mosquitoes can persist in the wild, the non-Wolbachia mosquitoes cannot dominate the population.

The results indicate that the introduction of Wolbachia-infected mosquitoes can be a powerful strategy if the Wolbachia-infected mosquitoes can dominate the population in the long term. This is because in dengue endemic countries, the dengue season lasts for more than 90-120 days, the approximate time duration in which the experiments on Wolbachia intervention have been conducted in far north Queensland $[2,16]$.

Future work stemming from this model can be conducted, such as including seasonality effects in the model. Several parameters such as the maturation rate, death rate and reproductive rate are temperature dependent [19], hence including seasonality effects could improve the model provided adequate data on the temperature dependence is available. By including seasonal variation in all temperature-dependent parameters, the model outcome would give detailed information about the mosquito population dynamics as temperature varies. This would aid the understanding of the effectiveness of this intervention, particularly in the areas where the temperature is extreme, such as Queensland.

Another interesting extension that can be undertaken is modelling the mosquito (Aedes aegypti) dispersal dynamics. The aim is to predict the areas that mosquitoes disperse to and their concentration, and hence determine the dengue endemic areas in which a Wolbachia intervention could work effectively. For this, distance and time are required and hence partial differential equations are needed. A travelling wave approach, coupled with the underlying population dynamics, would be both novel and interesting. It could be used to help predict where further releases of Wolbachiainfected mosquitoes would be most effective. 


\section{Acknowledgements}

Meksianis Z. Ndii acknowledges AusAID for providing him an Australian Development Scholarship (ADS) for studying at the Mathematical Sciences Institute, The Australian National University, and also The University of Newcastle, Australia, for a PhD scholarship. We would like to thank two anonymous reviewers for their constructive comments, which have helped to improve the paper.

\section{References}

[1] M. N. Burattini, M. Chen, A. Chow, F. A. B. Coutinho, K. T. Goh, L. F. Lopez, S. Ma and E. Massad, "Modelling the control strategies against dengue in Singapore", Epidemiol. Infect. 136 (2008) 309-319; doi:10.1017/S0950268807008667.

[2] A. A. Hoffmann et al., "Successful establishment of Wolbachia in Aedes populations to suppress dengue transmission", Nature 476 (2011) 454-457; doi:10.1038/nature10356.

[3] I. Iturbe-Ormaetxe, T. Walker and S. L. O'Neill, "Wolbachia and the biological control of mosquito-borne disease", EMBO Rep. 12 (2011) 508-518; doi:10.1038/embor.2011.84.

[4] R. Kongnuy, P. Pongsumpun and I.-M. Tang, "Mathematical model for dengue disease with maternal antibodies", Int. J. Biol. Life Sci. 7 (2011) 74-83.

[5] N. A. Maidana and H. M. Yang, "Describing the geographic spread of dengue disease by traveling waves", Math. Biosci. 215 (2008) 64-67; doi:10.1016/j.mbs.2008.05.008.

[6] S. Marino, I. B. Hogue, C. J. Ray and D. E. Kirschner, "A methodology for performing global uncertainty and sensitivity analysis in system biology", J. Theoret. Biol. 254 (2008) 178-196; doi:10.1016/j.jtbi.2008.04.011.

[7] C. J. McMeniman, R. V. Lane, N. B. Cass, A. W. C. Fong, M. Sidhu, Y.-F. Wang and S. L. O’Neill, "Stable introduction of a life-shortening Wolbachia infection into the mosquito Aedes aegpyti", Science 323 (2009) 141-144; doi:10.1126/science.1165326.

[8] I. R. Montella, A. J. Martins, P. F. Viana-Mendeiros, J. B. P. Lima, I. A. Braga and D. Valle, "Insecticide resistance mechanisms of Brazilian Aedes aegypti populations from 2001 to 2004", Am. J. Trop. Med. Hyg. 77 (2007) 467-477.

[9] J. G. Ordóñez-Gonzalez, R. Mercado-Hernandez, A. E. Flores-Suarez and I. Fernández-Salas, "The use of sticky ovitraps to estimate dispersal of Aedes aegypti in northeastern Mexico", J. Am. Mosq. Control Assoc. 17 (2001) 93-97.

[10] M. Otero, H. G. Solero and N. Schweigmann, "A stochastic population dynamics model for Aedes aegypti: formulation and application to a city with temperate climate", Bull. Math. Biol. 68 (2006) 1945-1974; doi:10.1007/s11538-006-9067-y.

[11] A. F. Read and M. B. Thomas, "Mosquitoes cut short", Science 323 (2009) 51-52; doi:10.1126/science. 1168659 .

[12] T. Ruang-areerate and P. Kittayapong, "Wolbachia transinfection in Aedes aegypti: a potential gene driver of dengue vectors", Proc. Nat. Acad. Sci 103 (2006) 12,534-12,539; doi:10.1073/pnas.0508879103.

[13] S. P. Sinkins, "Wolbachia and cytoplasmic incompatibility in mosquitoes", Insect Biochem. Molec. Biol. 34 (2004) 723-729; doi:10.1016/j.ibmb.2004.03.025.

[14] G. L. Swartzman and S. P. Kaluzny, Ecological simulation primer (Macmillan, New York, 1987).

[15] A. P. Turley, L. A. Moreira, S. L. O’Neill and E. A. McGraw, "Wolbachia infection reduces bloodfeeding success in the dengue fever mosquito, Aedes aegypti", PLoS Neglected Tropical Diseases 3 (2009) e516; doi:10.1371/journal.pntd.0000516.

[16] T. Walker et al., "The wMel Wolbachia strain blocks dengue and invades caged Aedes aegypti populations", Nature 476 (2011) 450-453; doi:10.1038/nature10355.

[17] World Health Organization, Dengue and severe dengue, Fact Sheet No. 117 (2002), http://www.who.int/mediacentre/factsheets/fs117/en/index.html. 
[18] Z. Xi, C. C. H. Khoo and S. L. Dobson, "Wolbachia establishment and invasion in an Aedes aegpyti laboratory population”, Science 310 (2005) 326-328; doi:10.1126/science.1117607.

[19] H. M. Yang, M. L. G. Macoris, K. C. Galvani, M. T. M. Andrighetti and D. M. V. Wanderley, "Assessing the effects of temperature on the population of Aedes aegypti, the vector of dengue", Epidemiol. Infect. 137 (2009) 1188-1202; doi:10.1017/S0950268809002040.

[20] H. L. Yeap et al., "Dynamics of the 'Popcorn' Wolbachia infection in outbred Aedes aegypti informs prospects for mosquito vector control”, Genetics 187 (2011) 583-595;

doi:10.1534/genetics.110.122390. 